Pathologe 2008 · [Suppl 2] 29:389-390

DOI 10.1007/s00292-008-1082-3

Online publiziert: 28. September 2008

c) Springer Medizin Verlag 2008

\author{
K. Goerttler \\ Pathologisches Institut, Universitätsklinikum Heidelberg
}

\title{
Kurt Wegener
}

\author{
26.9.1934-13.12.2007
}

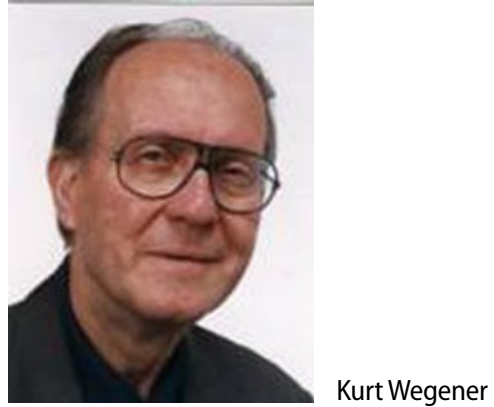

In der Nacht zum 13. Dezember 2007 verstarb nach jahrelanger schmerzhafter, in das Skelettsystem metastasierender maligner Neoplasie der Prostata Prof. Dr. Kurt Wegener, ehemaliger Chefarzt des Pathologischen Institutes der Städtischen Krankenanstalten in Ludwigshafen.

Es ist tragisch und ein Anachronismus zugleich, wenn ein Doktorvater einen Nachruf auf seinen Schüler verfassen muss, der sich noch dazu mit seiner zweiten Doktorandin vermählte.

Kurt Wegener war über fünf Jahrzehnte mein Doktorand, Mitarbeiter, Freund und lieber Gesprächspartner, beginnend in Kiel, später bis wenige Tage vor seinem Tod in Heidelberg.

Kurt Wegener wurde am 26. September 1934 als Sohn des Bürgermeisters Erich Wegener in Gotha/Thüringen geboren. Bis zu seiner Einschulung lebte er in Ohrdruff/Thüringen. 1947 erfolgten die Übersiedlung nach Wilhelmshaven und der Eintritt in das dortige naturwissenschaftliche Gymnasium, 1955 die Reifeprüfung.

Er begann das Medizinstudium ab 1955 bis zum Physikum an der ChristianAlbrechts-Universität in Kiel, bis zum Wintersemester 1957/58 an der Universität Hamburg, ab dem Wintersemester
1958 wieder in Kiel, dort legte er 1961 das medizinische Staatsexamen ab.

In Kiel trat Kurt Wegener in mein Leben. Als gerade habilitierter Privatdozent für Pathologie suchte ich und fand einen belastungsfähigen, kritischen, eminent fleißigen Doktoranden, der eine mit „sehr gut" bewertete Dissertation Über die experimentelle Trypanblauschädigung von Rattenembryonen während der Phase der Herzbildung verfasste. In Anerkennung seiner besonderen Leistung reichte ich Kurt Wegeners Arbeit beim Arch. für Kreislaufforschung ein und überlies ihm die alleinige Autorschaft. Durch die Abfassung in deutscher Sprache konnten die hervorragenden Ergebnisse der Studie leider den deutschen Sprachraum nicht überschreiten.

Kurt Wegener blieb mein wissenschaftlicher Mitarbeiter. Er prüfte mittels Mitosezählung an Hühnchenembryonen die Wachstumsaktivitäten verschiedener Abschnitte des embryonalen Hühnchenherzens; auch diese Ergebnisse wurden publiziert. Danach fand ich für ihn eine Assistentenstelle am Kölner Institut für medizinische Isotopenforschung unter Prof. Maurer. Kurt Wegener wurde Mitarbeiter der mit der Methode der Tritiummarkierung erfahrenen Frau Prof. Brigitte Maurer-Schulze.

Alles war angelegt auf eine weitere fruchtbare gemeinsame wissenschaftliche Tätigkeit, aber die Voraussetzungen änderten sich. 1964 nahm Kurt Wegener in Heidelberg eine ihm von Wilhelm Doerr angebotene Stelle an, wurde dessen Mitarbeiter und späterer Oberarzt, zusammen mit Uwe Bleyl. Er habilitierte sich 1967 mit einer Schrift Untersuchungen zur Pathogenese coronarsklerotischer Plaques, war am
Sonderforschungsbereich „Kardiovaskuläres System“ der Deutschen Forschungsgemeinschaft (DFG) beteiligt, wurde Oberarzt und nach der Facharztanerkennung „Leitender Oberarzt“.

Kurt Wegener lernte in dieser Zeit den späteren Radiologen Gerhard van Kaick kennen, der zum Deutschen Krebsforschungszentrum (DKFZ) in Heidelberg wechselte und am Thorotrast-Programm tätig war. Hieraus wurde eine langjährige freundschaftliche und wissenschaftliche Zusammenarbeit, wobei Kurt Wegener den Part des Pathologen übernahm. Dieses anspruchsvolle, in mehreren Zeitschriften publizierte Programm erfuhr weltweite Aufmerksamkeit.

1973 wurde Kurt Wegener zum außerplanmäßigen Professor der Universität ernannt und im gleichen Jahr zum Leiter des Institutes für Pathologie am Klinikum der Stadt Ludwigshafen berufen. Dort übernahm er von 1978-1982 auch die Funktion des Ärztlichen Direktors des Klinikums.

Kurt Wegener hat als profilierter Pathologe große Verdienste für die ärztliche Fortbildung in der gesamten angrenzenden Region erworben. Dabei halfen ihm über seine fachliche Kompetenz hinaus eine vornehme und verständnisvolle Art, verbunden mit Entscheidungskraft und Durchsetzungsfähigkeit. Kurt Wegener war bei Kollegen, Mitarbeitern und Pflegepersonal hochangesehen. Er gewann mit seiner sachlich-verständigen und gewinnenden Liebenswürdigkeit das Vertrauen von Klinikum und Stadtverwaltung.

Nach der Wiedervereinigung wurde Kurt Wegener Mitglied des Teilprojektes Pathologie der deutschen Uranerzbergarbeiterstudie, die mit Unterstützung des 
Bundesministeriums für Umwelt, Naturschutz und Reaktorsicherheit durchgeführt wurde. Die Ergebnisse wurden in anerkannten Zeitschriften veröffentlicht.

Nach 24 Dienstjahren schied Kurt Wegener 1997 auf eigenen Wunsch aus dem aktiven Dienst aus. Er wollte sich im Ruhestand seine Wünsche nach einer intensiveren Beschäftigung mit moderner Kunst, Lyrik (großer Verehrer von Gottfried Benn) und moderner Musik erfüllen. Er liebte die Literatur und das geschliffene Wort und damit speziell Lichtenberg, war Mitglied der Lichtenberg-Gesellschaft. In Heidelberg nahm er am Universitätsleben teil, besuchte Vorlesungen über Philosophie, wurde dank ,tatkräftiger und langfristiger Unterstützung “ Ehrenbürger der Ruperto-Carola.

Damit nicht genug: Schon 1985 wurde Kurt Wegener Mitglied des Vereins „Helfer ohne Grenzen“. Er organisierte Patenschaften, half Kriegswaisen, in seinem Namen wurde ein Fond für bosnische Medizinstudenten zur Erleichterung des Studiums eingerichtet.

Wenige Monate nach seinem Ausscheiden aus dem Dienst erreichte ihn die traurige Diagnose einer malignen Neoplasie der Prostata. Diese wurde über die gesamte folgende Lebenszeit mit allen modernen Verfahren therapiert, aber ein Fortschreiten war nicht aufzuhalten. Kurt Wegener nahm sein Schicksal mit bewundernswerter Stärke und in völliger geistiger Klarheit hin. Sein Tod war eine Erlösung.

Mit Kurt Wegener verlieren die deutschen Pathologen einen fachlich wie menschlich hervorragenden Vertreter ihres Faches. Wer ihm näher treten durfte, trauert um einen lieben, humorvollen Freund, einen belesenen und erfahrenen Kunstkenner und einen Wohltäter für Notleidende.

Unser Mitgefühl gilt auch seiner lieben Frau.

Klaus Goerttler, Heidelberg

\section{Korrespondenzadresse}

Prof. Dr. K. Goerttler

Pathologisches Institut, Universitätsklinikum Heidelberg

Im Neuenheimer Feld 220, 69120 Heidelberg

Klaus.Goerttler@med.uni-heidelberg.de

\section{Rudolf-Virchow-Preis}

\author{
Ausschreibung 2009
}

Der Preis von 5.000 Euro wird laut Satzung der Rudolf-Virchow-Stiftung für Pathologie einem Pathologen unter 40 Jahren für eine noch nicht veröffentlichte oder eine nicht länger als ein Jahr vor der Bewerbung publizierte wissenschaftliche Arbeit verliehen.

Die Verleihung des Preises erfolgt auf der 93. Tagung der Deutschen

Gesellschaft für Pathologie e.V. 2009.

Zusammen mit einem Lebenslauf und einer Publikationsliste reichen Bewerber ihre Arbeit ein (bitte alle Unterlagen in doppelter Ausfertigung!).

Abgabetermin: bis 31. Dezember 2008

Einzureichen bei:

Geschäftsstelle der Deutschen Gesellschaft für Pathologie e.V. c/o Institut für Pathologie der MH-Hannover

Carl-Neuberg-Str. 1

30625Hannover

Die Satzung der Rudolf-Virchow-Stiftung für Pathologie sowie weitere Informationen zum Rudolf-Virchow-Preis finden Sie unter http.//www.mh-hannover.de/institute/pathologie/dgp.

Deutsche Gesellschaft für Pathologie e.V

-Vorstand- Frühjahr 2008 